Nuran Asan, email: kelesnuran@gmail.com Guzide Dogan, email guzidedogan@gmail.com Sadi Turkay, Email: saturkay@gmail.com Meki Bilici, Dicle University, Department of Pediatric Cardiology, Diyarbakır, Turkey, Email: drmekibilici@hotmail.com

Corresponding Author name: Güzide Doğan Mailing address: Hafsa Sutan Mh. 4801 Sk. 5/26 Manisa/ Turkey E-mail: guzidedogan@gmail.com

\section{The Relationships of Cardiac Structures and Functions with Adiponectin, C-Reactive Protein, and Interleukin-6 Levels in Obese Children}

Nuran Asan, Guzide Dogan, Sadi Turkay, Meki Bilici, Nesibe Andiran, Cemile Koca.

Objective: Obesity in childhood is associated with increased morbidity and mortality in adulthood due to cardiovascular disease. C-Reactive protein (CRP) and interleukin-6 (IL-6) levels are elevated in obese patients, whereas the adiponectin level is negatively correlated. This study was conducted to determine the effects of obesity on cardiac structure and functions and to investigate the relationship of cardiac structures and functions with CRP, II-6, and adiponectin levels.

Patients and Methods: A total of 38 obese and 30 healthy non-obese children were included. The anthropometric profiles of all the children were recorded. CRP, IL-6, adiponectin, and fasting glucose insulin levels were measured. The Homeostatic Model Assessment of Insulin Resistance (HOMA-IR) was calculated. The children were assessed using conventional transthoracic echocardiography and tissue Doppler imaging.

Results: Serum CRP, IL-6, insulin, and HOMA-IR were higher, and adiponectin levels were lower in obese subjects. Left ventricular wall thickness and dimensions, and left atrial diameter were greater in the obese group and the myocardial performance index (MPI) was measured higher $(P=0.001)$. These alterations of cardiac structure were found to be positively correlated with body mass index (BMI), waist circumference, insulin and HOMA-IR, and negatively correlated with adiponectin.

Conclusion: These alterations in cardiac structure and functions in obese children indicate cardiovascular diseases in adulthood, and can be measured by Doppler echocardiography. In addition, BMI, waist circumference, insulin, and HOMA-IR are useful parameters for both cardiac structure and cardiac functions. Adiponectin, should also be considered as a new parameter for cardiac structure.

Keywords: cardiac functions, obesity, childhood, HOMA-IR, adiponectin, interleukin-6

\section{INTRODUCTION}

Nowadays, obesity is one of the most important public health problems. Cardiac problems secondary to obesity are mostly seen as clinically silent diseases. Physiopathological changes occur before any clinically apparent findings $[1,2]$. Diseases related to vascular damage, such as myocardial infarction and stroke are late clinical results of obesity. If only hereditary and environmental factors (eg, dietary habits, low energy consumption and psychosocial factors) play a role in the pathogenesis and there is no evidence of underlying systemic disorders, then it is known as simple obesity. Secondary obesity is defined as obesity due to endocrinopathies, central nervous system (CNS) disease, drugs and some genetic diseases [3, 4]. The most effective treatment for obesity is the prevention of underlying pathogenetic causes.

The aim of this study was to first investigate the effects of obesity on cardiac structure and function, which result in fatal conditions such as coronary heart disease, hypertension, dyslipidemia, insulin resistance and diabetes 
mellitus (DM), and secondly to evaluate any correlation between echocardiographic parameters and blood levels of adiponectin, interleukin-6 (IL-6) and C-reactive protein (CRP) in the early diagnosis of cardiac dysfunction.

\section{PATIENTS AND METHODS}

The study was performed on 38 children aged between 6-16 years, diagnosed with simple obesity in the Pediatric Endocrinology and Pediatrics Clinics. A control group was formed of 30 age and gender-matched healthy children. A detailed history of all the children was recorded and physical examination was performed at the baseline evaluation. According to the Body Mass Index (BMI) percentiles for the Turkish population based on gender and age, obesity was defined as a BMI at or above the $95^{\text {th }}$ percentile [5].The height and weight of the parents were also recorded within the familial history taking. The children with secondary obesity, congenital or acquired heart disease, hypertension, or using any drugs affecting the lipid or glucose metabolism or cardiovascular function were excluded from the study.

The study protocol was approved by the local Ethics Committee and conducted in accordance with the principles of the Helsinki Declaration. Written informed consent was obtained from the parents or legal guardians of all study participants.

\section{Measurements}

Blood pressure (BP) was measured while the patient was sitting, following a 10-minute rest period, by an age-appropriate cuff covering two thirds of the left forearm. Children were only included if systolic and diastolic pressures were below the 95th age and gender-adjusted percentile. Heart rate was recorded at the examination for all subjects. Body mass index was calculated using the following formula: $\mathrm{BMI}=$ Body Weight $(\mathrm{kg}) /$ height $^{2}$ (m). Blood samples were taken at 8:00-9:00 am following a minimum 12-hours fasting. Serum adiponectin and IL-6 levels were studied using enzyme-linked immunosorbent assay (ELISA). Serum CRP concentrations were measured with the nephelometric procedure. Enzymatic colorimetric tests were used for the measurement of other biochemical analyses. The homeostasis model assessment of insulin resistance (HOMA-IR), as the index of insulin sensitivity, was determined using the following formula:
Blood Glucose (mmol/L) x Insulin (mU/L)

HOMA-IR:

The value above 3.16 was the cutoff point of HOMA-IR to define insulin resistance [6]. Echocardiography was performed using a Philips MCMD02AA model two-dimensional color echocardiography device with M-mode, pulse wave Doppler and tissue Doppler capabilities with a 1.9-5 MHz phased array transducer. The echocardiographic evaluations were performed in both groups by the same doctor.

\section{Statistical Analysis}

The SPSS 13 software package (Statistical Package for the Social Sciences, Chicago, IL) was used for statistical data evaluation. All results were expressed as mean \pm standard deviation (SD). Categorical variables were compared using the Chi-squared test. The Kolmogorov-Smirnov test was applied to evaluate goodness of fit to normal distribution. Normally distributed continuous variables were compared between the study and control groups using a parametric test (Student's t-test) or a non-parametric test (Mann-Whitney U), depending, respectively, on normal distribution or not. The Pearson test for linear correlation was applied to investigate correlations between pairs of continuous variables. A value of $\mathrm{p}<0.05$ was accepted as statistically significant.

\section{RESULTS}

A total of 68 children, comprising 38 obese and 30 control subjects were included in the study. No statistically significant difference was determined between the obese and control groups in respect of age and gender (Table 1). The respective values for weight were $66.1 \pm 21.3 \mathrm{~kg}$ and $38.5 \pm 11.4 \mathrm{~kg}$, and for height $152.3 \pm 13.9 \mathrm{~cm}$ and $142.8 \pm 14.6 \mathrm{~cm}$, with differences that were statistically significant $(P=0.001$ and $P=0.008$, respectively). The BMI of the obese group was $27.6 \pm 4.4$ and that of the control group, $18.4 \pm 2.3 \mathrm{~kg} / \mathrm{m}^{2}$. The waist circumference measurements were $90.9 \pm 12.2 \mathrm{~cm}$ and $64.8 \pm 7.9 \mathrm{~cm}$, respectively. The differences between these variables were found to be statistically significant $(P=0.001)$. The demographic characteristics of the study group are shown in Table 1. 
Cardiac Structures and Functions, Adiponectin, CRP and IL-6 in Obese Children

Table 1. Demographic Characteristics of the Groups

\begin{tabular}{|c|c|c|c|}
\hline & $\begin{array}{l}\text { Obese group } \\
(n=38)\end{array}$ & $\begin{array}{c}\text { Control group } \\
(n=30)\end{array}$ & $P$ value \\
\hline Age (years) & $11.5 \pm 2.7$ & $10.7 \pm 2.4$ & 0.20 \\
\hline Gender (male/female) & $20 / 18$ & $13 / 17$ & 0.60 \\
\hline Height (cm) & $152.3 \pm 13.9 *$ & $142.8 \pm 14.6$ & $<0.05$ \\
\hline Height z score & $0.29 \pm 0.91 *$ & $-0.32 \pm 0.95$ & $<0.05$ \\
\hline Waist Circumference $(\mathrm{cm})$ & $90.9 \pm 12.2^{*}$ & $64.8 \pm 7.9$ & $<0.05$ \\
\hline Waist Circumference z score & $0.71 \pm 0.73^{*}$ & $-0.85 \pm 0.47$ & $<0.05$ \\
\hline BMI $\left(\mathrm{kg} / \mathrm{m}^{2}\right)$ & $27.6 \pm 4.4 *$ & $18.4 \pm 2.3$ & $<0.05$ \\
\hline BMI z score & $0.70 \pm 0.75^{*}$ & $-0.88 \pm 0.39$ & $<0.05$ \\
\hline
\end{tabular}

BMI: Body Mass Index, BP: Blood pressure, ${ }^{*} \boldsymbol{P}<0.05$

\section{Laboratory Findings}

The fasting blood glucose (FBG) mean levels were similar in the study and control groups $(P=0.70)$. The mean insulin levels were significantly higher in the obese group than in the control group $(P=0.001)$. The HOMA-IR values were significantly higher in the obese children $(P=0.001)$. The CRP and IL-6 levels were significantly higher in the obese children compared to the control group. The adiponectin serum levels were $13.01 \pm 4.7 \mu \mathrm{g} / \mathrm{mL}$ in the obese group and $18.3 \pm 6.2 \mu \mathrm{g} / \mathrm{mL}$ in the control group and and were determined as significantly lower in the obese children (Table 2).

Table 2. Laboratory Values of the Groups

\begin{tabular}{|c|c|c|c|}
\hline & $\begin{array}{l}\text { Obese group } \\
\qquad(n=38)\end{array}$ & $\begin{array}{l}\text { Control group } \\
\qquad(n=30)\end{array}$ & $P$ value \\
\hline Glucose, mg/dL & $88.3 \pm 5.6$ & $89.1 \pm 7.8$ & 0.70 \\
\hline Insulin, $\mu \mathrm{lu} / \mathrm{mL}$ & $15.2 \pm 8.5^{*}$ & $6.6 \pm 4.1$ & 0.001 \\
\hline HOMA-IR & $3.3 \pm 1.9 *$ & $1.5 \pm 1.0$ & 0.001 \\
\hline CRP, mg/L & $3.6 \pm 1.7^{*}$ & $2.7 \pm 1.9$ & 0.02 \\
\hline IL-6, pg/mL & $2.6 \pm 1.2^{*}$ & $2.1 \pm 1.5$ & 0.001 \\
\hline Adiponectin, $\mu \mathrm{g} / \mathrm{mL}$ & $13.1 \pm 4.7^{*}$ & $18.3 \pm 6.2$ & 0.001 \\
\hline $\mathrm{TC}, \mathrm{mg} / \mathrm{dL}$ & $157.8 \pm 30.4$ & $153.8 \pm 28.5$ & 0.60 \\
\hline $\mathrm{HDL}, \mathrm{mg} / \mathrm{dL}$ & $41.7 \pm 10.4^{*}$ & $55.8 \pm 16.3$ & 0.001 \\
\hline $\mathrm{LDL}, \mathrm{mg} / \mathrm{dL}$ & $92.5 \pm 23.3$ & $82.5 \pm 30.5$ & 0.10 \\
\hline VLDL, mg/dL & $22.4 \pm 13.1^{*}$ & $15.5 \pm 15.1$ & 0.001 \\
\hline $\mathrm{TG}, \mathrm{mg} / \mathrm{dL}$ & $113.4 \pm 66.7^{*}$ & $63.9 \pm 26.1$ & 0.001 \\
\hline
\end{tabular}

CRP: C-reactive protein, HDL: High-density lipoprotein, HOMA-IR: Homeostasis model assessment of insulin resistance, IL-6: Interleukin-6, LDL: Low-density lipoprotein, TC: Total cholesterol, TG: Triglycerides, VLDL: Very low-density lipoprotein

\section{Echocardiographic Findings}

\section{Cardiac Structure}

When examining the echocardiographic parameters, Left ventricular internal diameter end diastole (LVIDd) and end systole (LVIDs) were found to be higher in the obese children than in the control group ( $P=0.017, P=0.025$, respectively). The enddiastolic interventricular septum diameter (IVSd) was higher $(P=0.029)$, as were both the left atrial (LA) and the left ventricular posterior wall diastolic diameters (LVPWd) $(P=0.001, P=0.001)$ in the obese group (Table 3).

Table 3. Echocardiographic Findings of Cardiac Structures

\begin{tabular}{lccc}
\hline & $\begin{array}{c}\text { Obese group } \\
(\mathbf{n = 3 8 )}\end{array}$ & $\begin{array}{c}\text { Control group } \\
(\mathbf{n = 3 0 )}\end{array}$ & P value \\
\hline LVIDd, cm & $4.38 \pm 0.50^{*}$ & $4.03 \pm 0.57$ & $\mathbf{0 . 0 1 7}$ \\
LVIDs, cm & $2.88 \pm 0.42^{*}$ & $2.61 \pm 0.39$ & $\mathbf{0 . 0 2 5}$ \\
IVSd, cm & $0.78 \pm 0.18^{*}$ & $0.69 \pm 0.15$ & $\mathbf{0 . 0 2 9}$ \\
IVSs, cm & $1.08 \pm 0.21$ & $1.00 \pm 0.25$ & 0.08 \\
LVPWd, cm & $0.71 \pm 0.19^{*}$ & $0.54 \pm 0.14$ & $\mathbf{0 . 0 0 1}$ \\
LVPWs, cm & $1.04 \pm 0.21$ & $0.97 \pm 0.20$ & 0.21 \\
LA Diameter, cm & $3.53 \pm 3.15^{*}$ & $2.62 \pm 0.34$ & $\mathbf{0 . 0 0 1}$ \\
\hline
\end{tabular}

IVSd: Interventricular septum diastolic diameter, IVSs: Interventricular septum systolic diameter, LA: Left atrium, LA/Ao: Left atrial to aortic root ratio LIVDd: Left ventricular end-diastolic diameter, LIVDs: Left ventricular end-systolic diameter, LVPWd: Left ventricular diastolic posterior wall thickness, LVPWs: Left ventricular systolic posterior wall thickness, ${ }^{*} P<0.05$

\section{Cardiac Functions}

The parameters used to evaluate systolic and diastolic functions in both the obese and control groups are shown in Table 4. The mean myocardial performance index (MPI) values were $0.51 \pm$ 0.05 in the obese group and $0.40 \pm 0.04$ in the control group. This difference was statistically significant. 


\begin{tabular}{lccc}
\hline Table 4. Echocardiographic Findings on Cardiac Function & \\
\hline & $\begin{array}{c}\text { Obese group } \\
(\mathbf{n}=\mathbf{3 8})\end{array}$ & $\begin{array}{c}\text { Control group } \\
(\mathbf{n}=\mathbf{3 0})\end{array}$ & $\boldsymbol{P}$ value \\
\hline LVEF, \% & $63 \pm 11$ & $67 \pm 6$ & 0.060 \\
\hline FS, \% & $36 \pm 7$ & $37 \pm 5$ & 0.238 \\
\hline E wave, cm/sn & $93 \pm 13$ & $96 \pm 20$ & 0.700 \\
\hline A wave, cm/sn & $47 \pm 14$ & $48 \pm 16$ & 0.680 \\
\hline E/A ratio & $2.0 \pm 0.4$ & $2.1 \pm 0.4$ & 0.285 \\
\hline IVCT, msn & $52 \pm 10^{*}$ & $48 \pm 10$ & $\mathbf{0 . 0 0 9}$ \\
\hline IVRT, msn & $78 \pm 13^{*}$ & $70 \pm 8$ & $\mathbf{0 . 0 0 5}$ \\
\hline LVET, msn & $254 \pm 35$ & $268 \pm 26$ & 0.061 \\
\hline MPI & $0.51 \pm 0.05^{*}$ & $0.40 \pm 0.04$ & $\mathbf{0 . 0 0 1}$ \\
\hline
\end{tabular}

FS: Fractional shortening, IVCT: Isovolumetric contraction time, IVRT: Isovolumetric relaxation time, LVEF: Left ventricular ejection fraction, LVET: Left ventricular ejection time, MPI: Myocardial performance index. ${ }^{*} P<0.05$

\section{Correlations}

A statistically significant correlation was found between BMI and the measurements for cardiac structure: LVIDs $(r=0.523$, $P=0.001)$, LVIDd $(\mathrm{r}=0.520, \mathrm{P}=0.001)$, IVSd $(\mathrm{r}=0.366, P=0.020)$, and LVPWd ( $r=0.403, P=0.010)$. Waist circumference, one of the most reliable indexes of abdominal obesity, was positively correlated with LVIDs $(r=0.562, P=0.001)$, LVIDd $(r=0.531$, $P=0.001)$ and LVPWd $(r=0.369, P=0.016)$. There was also a significant, direct correlation between MPI, a measure of both diastolic and systolic function, and both BMI and waist circumference ( $\mathrm{r}=0.352, P=0.021$ and $\mathrm{r}=0.355, P=0.018$, respectively).

The HOMA-IR value, used as an index of insulin sensitivity, was observed to be positively and significantly correlated with LVIDs and LVIDd. No relationship was determined between HOMA-IR and either septum or posterior wall thickness. Significant and direct relationships were shown for MPI, an index of cardiac function, with both insulin and HOMA-IR (Table 5). The study of lipid profile in relation to cardiac structures yielded no significant correlations of the blood lipid profile with LVIDd, LVIDs, IVSd and LVPWd values. No significant correlation was found between MPI and the blood lipid values (Table 5).

Table 5. Correlations of Cardiac Structures and MPI with Insulin, HOMA-IR and Lipid Profile

\begin{tabular}{|c|c|c|c|c|c|c|c|c|}
\hline \multirow{2}{*}{ LVIDd } & $r$ & 0.481 & 0.484 & 0.199 & 0.041 & -0.265 & 0.145 & 0.126 \\
\hline & $p$ & $0.001 *$ & $0.001 *$ & 0.104 & 0.738 & 0.229 & 0.238 & 0.307 \\
\hline \multirow{2}{*}{ LVIDs } & $r$ & 0.560 & 0.583 & 0.231 & 0.119 & -0.202 & 0.109 & 0.189 \\
\hline & $p$ & $0.001 *$ & $0.001^{*}$ & 0.160 & 0.332 & 0.100 & 0.377 & 0.122 \\
\hline \multirow{2}{*}{ LVPWd } & $r$ & 0.259 & 0.230 & 0.183 & -0.018 & -0.188 & 0.173 & 0.056 \\
\hline & $p$ & 0.110 & 0.126 & 0.301 & 0.771 & 0.425 & 0.159 & 0.351 \\
\hline \multirow{2}{*}{ MPI } & $r$ & 0.598 & 0.602 & 0.240 & 0.137 & -0.401 & 0.224 & 0.249 \\
\hline & $p$ & $0.001 *$ & $0.001 *$ & 0.071 & 0.266 & 0.090 & 0.066 & 0.200 \\
\hline
\end{tabular}

HDL: High-density lipoprotein, HOMA-IR: Homeostasis model assessment of insulin resistance, IVSd: Interventricular septum diastolic diameter, LDL: Low-density lipoprotein, LIVDd: Left ventricular end-diastolic diameter, LIVDs: Left ventricular end-systolic diameter, LVPWd: Left ventricular diastolic posterior wall thickness, MPI: Myocardial performance index, r: Pearson's correlation coefficient TC: Total cholesterol, VLDL: Very low-density lipoprotein, TG: Triglycerides * $\mathrm{p}<0.05$

\section{Correlations of Cardiac Structure and Function Measurements with Inflammatory Markers}

LVIDd was the only structural cardiac variable with a statistically significant reverse correlation with the adiponectin level $(r=-0.370$, $P=0.02)$. LVIDs, IVSd or LVPWd were not correlated with adiponectin levels. MPI, which is used to evaluate cardiac function $(\mathrm{r}=-0.19, P=0.20)$, was not determined to be correlated with adiponectin levels. The structural cardiac variables LVIDs, LVIDd, IVSd or LVPWd did not show a significant correlation with CRP. No correlation was determined between CRP and MPI. There was no correlation observed between LVIDs, LVIDd, IVSd, LVPWd or MPI and IL-6 levels.

\section{DISCUSSION}

While BP was within normal limits for both groups, the systolic and diastolic pressure in the obese group was statistically higher than in the control group. This could be interpreted as a risk factor for the development of hypertension later in life. A significant relationship has been shown to exist between abdominal circumference and BP. The frequency of hypertension is increased especially with abdominal obesity [7]. Ma et al. reported that waist circumference is an indicator of cardiovascular risk factors [8]. Similar to reports in literature, the results of the current study showed a statistically significant correlation between waist circumference and both systolic and diastolic BP. While the obese 
subjects in this study had higher very low-density lipoprotein (VLDL) and triglycerides (TG), and lower high-density lipoprotein (HDL) levels than the control subjects, no difference was observed in the total cholesterol (TC) and low-density lipoprotein (LDL) values. A strong correlation with insulin resistance of dyslipidemias, especially high TG or low HDL levels, has also been reported in published studies [9].

Hyperinsulinemia and insulin resistance lead to this condition by causing an increase in hepatic VLDL synthesis and a reduction of lipase activity in peripheral tissues [10]. In a study by Weiss et al., a significantly lower HDL and higher TG level was reported in 439 obese children compared to the control group, while no such difference was present for LDL [11]. These small differences in the lipid profile may be related to the degree of obesity, the body composition and especially to the age and gender of the subjects, considering the available knowledge of the pronounced effect of sex hormones on the lipid profile [10].

There has been reported to be a strong epidemiological correlation between obesity and early-onset type $2 \mathrm{DM}$. Insulin resistance is recognized as an important risk factor for DM (9). HOMA-IR is often used to evaluate the level of insulin resistance [6]. Odelye et al reported that FBG levels were linked to weight gain in later years for Indian children aged 5-9 years [12]. In a study of 26 obese children and 36 normal-weight control subjects, aged 14 to 18 years, Vikram et al. found higher values of both insulin and HOMA-IR in the obese group and these values were also correlated with BMI and waist circumference [13]. Similarly, the obese group in the current study showed higher fasting insulin levels and HOMA-IR values compared to the control group. The mean HOMA-IR value in the current study group was above the cut-off level for insulin resistance in Turkish children, as defined by Keskin et al. [6]. The probable mechanism of insulin resistance in obese children may be explained by morphological and physiological changes in the increasing fatty tissue. One of these changes is seen in the secretion of pro-inflammatory cytokines (IL-6, Tumor necrosis factor- $\alpha$ (TNF- $\alpha$ )) which increases insulin resistance and reduces the signal intensity to insulin receptors [14]. Obesity causes mild inflammation, a process in which C-reactive protein, an acute phase reactant, plays an important role. A strong relationship between IL- 6 and obesity has been reported, and it is thought to have a possible role as a marker for coronary artery disease and type $2 \mathrm{DM}$ through the stimulation of CRP and other acute-phase proteins [15]. In a study by Gøbel et al. comparing healthy children with increased CRP levels and control children with normal CRP, a relationship was reported between increased CRP levels and a clinical and biochemical atherogenic lipid profile [16]. The same study also indicated a correlation between CRP and IL- 6 concentrations. In another study by Hura et al investigating correlations between CRP, obesity, $\mathrm{BP}$ and serum lipid levels in 86 obese and 58 normal boys with a mean age of 11.2 years, CRP levels were found to be elevated in the obese children [17]. Furthermore, a similarly direct correlation of CRP was observed with body weight, systolic and diastolic $\mathrm{BP}$ and the LDL/HDL ratio. The current study findings of elevated
CRP and IL-6 levels were in line with the literature. This elevation is a consequence of the increase in fat tissue in obesity, especially in visceral fat, which increases IL-6 secretion, stimulating hepatic CRP synthesis.

Iacobellis et al. evaluated cardiac morphology and function of 75 obese and 60 control subjects [18]. The obese group had increased left ventricular (LA) diameter, A-wave, left ventricular ejection fraction (LVEF), fractional shortening (FS) and isovolumetric relaxation time (IVRT), and reduced E-wave and A/E ratio, without any difference in LVIDd, LVIDs, IVS or LVPWd measurements. It was also observed that there was an increase in left ventricular mass, along with a decrease in E-wave and E/A ratio with age. A direct correlation was reported between BMI and left ventricular mass, and a weak but significant relationship was determined between insulin levels and either IVRT or E/A ratio. MPI, calculated by the conventional method, was found to be higher in adolescent obese subjects than in healthy controls by Levent et al. [19]. That study also compared hypertensive to normotensive adolescent obese subjects in respect of MPI and found a significantly higher value in the hypertensive group.

An increase in LVIDd, LVIDs, IVSd, LVPWd and LA measurements were observed in the current study echocardiographic evaluation of obese subjects. These values were also positively correlated with BMI and waist circumference. In addition, insulin levels and HOMA-IR were correlated with LVIDd and LVIDs, which was consistent with findings in literatures. No relationship was observed between the various structural cardiac measurements on one side and the inflammation markers CRP or IL-6. A review of the literature on the latter topic yields few reports. In one of these, by Malavazos et al., in 27 adult, normotensive obese subjects, higher CRP and IL-6 levels were determined compared to the control group, as well as a relationship between CRP and IL-6 on one side and LVPWd and IVSd on the other [20]. A study of adult subjects by Ebinç et al. found an inverse correlation between anti-inflammatory adiponectin levels with left ventricular mass, insulin levels and HOMA-IR values [21]. The effect of adiponectin on the left ventricular mass was explained in the same study by its inhibition of hypertrophic signal transmission and of protein synthesis in cardiomyocytes. Structural cardiac changes secondary to obesity have been documented by numerous published reports preceding the current study. These changes have been mainly observed in the left ventricular diameter and wall thickness [22, 23]. Different results might be due to differences in the subject's age, puberty status, duration of obesity and different levels of insulin resistance. The probable factors involved in cardiac structural change caused by obesity might be listed as hemodynamic modifications due to the increase in total blood volume, the ensuing compensatory mechanisms, hyperinsulinemia and insulin resistance. The growth-stimulating role and the regulatory effect on intracellular / extracellular calcium balance of insulin are both well known [24]. A similar role can be discussed with regard to the E-wave, an index of diastolic functions, the A wave, and the E/A ratio. Even though several published studies have reported a decrease in the $\mathrm{E}$-wave, an increase in the $\mathrm{A}$-wave or a decrease 
of the E/A ratio, other papers, including the current study, have shown no such change. The fact that the E/A ratio depends on age, respiratory rate and heart rate could be significant in such differences in the findings [19]. In respect of systolic function, most publications have generally observed that they were preserved. The current study also confirmed that there was no difference between the groups in respect of systolic function values such as LVEF or FS. The tissue Doppler echocardiographic investigation of the current study revealed higher isovolumetric contraction time (IVCT), IVRT and MPI values in obese children. MPI reflects both the systolic and diastolic function of the left ventricle. A previous study of adult obese subjects reported that MPI was correlated positively with the levels of the inflammation markers CRP and IL-6 [20]. No such relationship could be established in the current study. Moreover, no correlation was observed between MPI and adiponectin, which is known to be decreased in obesity and has an antiinflammatory action. To the best of our knowledge, the current study is the first in which the relationship between cardiac structure and function on one side and CRP, IL- 6 and adiponectin on the other has been investigated.

\section{Conclusions}

Obesity has a negative effect on the cardiovascular system, through mechanisms such as increased BP, dyslipidemia and insulin resistance. The inflammatory process secondary to the increase in fat tissue and reduction of adiponectin levels, a cardioprotective hormone, further contribute to this process. Obesity also affects the heart structure and function in children. Echocardiography is a non-invasive and useful method to characterize these changes, which are a precursor of cardiovascular disease in adulthood. BMI, waist circumference, insulin levels, HOMA-IR and adiponectin are parameters that are related to cardiac morphology, while BMI, waist circumference, insulin levels, and HOMA-IR are related to systolic and diastolic function, with regard to the cardiovascular condition of obese subjects. The first step in reducing the possible cardiovascular risk, in view also of the increasing prevalence of childhood obesity, is weight control. It is necessary to remain aware of cardiovascular complications related to obesity and to have a good knowledge of the complications of those subjects who have increased BMI, waist circumference, insulin levels, HOMA-IR values and reduced adiponectin levels.

Declaration of Conflicting Interests: The authors declare that they have no conflict of interest.

Financial Disclosure: No financial support was received.

\section{REFERENCES}

1. Poirier P, Giles TD, Bray GA, et al. Obesity and Cardiovascular Disease: Pathophysiology, Evaluation, and Effect of Weight Loss. Circulation. 2006;113:898-918.

2. Ogden CL, Carroll MD, Curtin LR, McDowell MA, Tabak CJ, Flegal KM. Prevalence of overweight and obesity in the United States 1999-2004. JAMA. 2006;295:1549-55.
3. Gurnani M, Birken C, Hamilton J. Childhood Obesity: Causes, Consequences, and Management. Pediatr Clin North Am. 2015;62:82140.

4. Viner R, Nicholls D. Managing obesity in secondary care: a personal practice. Arch Dis Child. 2005;90:385-90.

5. Wang Y, Wang JQ. A comparison of international references for the assessment of child and adolescent overweight and obesity in different populations. Eur J Clin Nutr. 2002;56:973-82.

6. Keskin M, Kurtoglu S, Kendirci M, Atabek ME, Yazici C. Homeostasis model assessment is more reliable than the fasting glucose/insulin ratio and quantitative insulin sensitivity check index for assessing insulin resistance among obese children and adolescents. Pediatrics. 2005; $115: 500-3$.

7. Macumber IR, Weiss NS, Halbach SM, Hanevold CD, Flynn JT. The Association of Pediatric Obesity With Nocturnal Non-Dipping on 24Hour Ambulatory Blood PressureMonitoring. Am J Hypertens. 2016;29:647-52.

8. Ma L, Cai L, Deng L, et al. Waist circumference is better than other anthropometric indices for predicting cardiovascular disease risk factors in Chinese Children -a Cross-Sectional Study in Guangzhou. J Atheroscler Thromb. 2016;23:320-9.

9. Boodai SA, Cherry LM, Sattar NA, Reilly JJ. Prevalence of cardiometabolic risk factors and metabolic syndrome in obese Kuwaiti adolescents. Diabetes Metab Syndr Obes. 2014;24:505-11.

10. Okada T, Saito E, Kuromori Y, et al. Relationship between serum adiponectin level and lipid composition in each lipoprotein fraction in adolescent children. Atherosclerosis. 2006;188:179-83.

11. Weiss R, Dziura J, Burgert TS, et al. Obesity and the metabolic syndrome in children and adolescents. N Engl J Med. 2004;350:2362-74.

12. Odeleye OE, de Courten M, Pettitt DJ, Ravussin E. Fasting hyperinsulinemia is a predictor of increased body weight gain and obesity in Pima Indian children. Diabetes. 1997;46:1341-5.

13. Vikram NK, Misra A, Pandey RM, Dwivedi M, Luthra K. Adiponectin, insulin resistance, and C-reactive protein in postpubertal Asian Indian adolescents. Metabolism. 2004;53:1336-41.

14. Martin LJ, Woo JG, Daniels SR, Goodman E, Dolan LM. The relationships of adiponectin with insulin and lipids are strengthened with increasing adiposity. J Clin Endocrinol Metab. 2005;90:4255- 9.

15. Berg AH, Scherer PE. Adipose tissue, inflammation, and cardiovascular disease. Circ Res. 2005;96:939-49.

16. Gøbel RJ, Jensen SM, Frøkiaer H, Mølgaard C, Michaelsen KF. Obesity, inflammation and metabolic syndrome in Danish adolescents. Acta Paediatr. 2012;101:192-200.

17. Hiura M, Kikuchi T, Nagasaki K, Uchiyama M. Elevation of serum $\mathrm{C}$-reactive protein levels is associated with obesity in boys. Hypertens Res. 2003;26:541-6.

18. Iacobellis G, Ribaudo MC, Leto G, et al. Influence of excess fat on cardiac morphology and function: study in uncomplicated obesity. Obes Res. 2002;10:767-73.

19. Levent E, Goksen D, Ozyurek AR, Darcan S, Coker M. Usefulness of the myocardial performance index (MPI) for assessing ventricular function in obese pediatric patients. Turk J Pediatr. 2005;47:34-8.

20. Malavazos AE, Corsi MM, Ermetici F, et al. Proinflammatory cytokines and cardiac abnormalities in uncomplicated obesity: relationship with abdominal fat deposition. Nutr Metab Cardiovasc Dis. 2007;17:294-302.

21. Ebinc H, Ebinc FA, Ozkurt ZN, et al. Impact of adiponectin on left ventricular mass index in non-complicated obese subjects. Endocr J. 2008;55:523-8

22. Korkmaz O, Gursu HA, Karagun BS. Comparison of echocardiographic findings with laboratory parameters in obese children. Cardiol Young. 2016;26:1060-5. 


\section{Cardiac Structures and Functions, Adiponectin, CRP and IL-6 in Obese Children}

23. Kibar AE, Pac FA, Ece İ, et al. Effect of obesity on left ventricular longitudinal myocardial strain by speckle tracking echocardiography in children and adolescents. Balkan Med J. 2015;32:56-63.
24. Chinali M, de Simone G, Roman MJ, et al. Impact of obesity on cardiac geometry and function in a population of adolescents: the Strong Heart Study. J Am Coll Cardiol. 2006;47:2267-73. 